\title{
Explosion driven Interfacial instabilities of granular media
}

\author{
KUN XUE ${ }^{1}$, Xiaoliang Shi ${ }^{2}$, Junsheng Zeng ${ }^{3}$, Baolin Tian ${ }^{4}$, Panpan Han $^{2}$, Jiarui Li ${ }^{2}$, Lan \\ $\mathrm{Liu}^{2}$, Baoqing Meng ${ }^{4}$ Xiaohu $\mathrm{Guo}^{5}$, and Chunhua Bai ${ }^{2}$ \\ ${ }^{1}$ Affiliation not available \\ ${ }^{2}$ Beijing Institute of Technology \\ ${ }^{3}$ Peking University \\ ${ }^{4}$ Institute of Applied Physics and Computational Mathematics \\ ${ }^{5}$ Science and Technology Facilities Council Daresbury Laboratory
}

April 28, 2020

\begin{abstract}
In this work, we investigate the evolution of a Richtmyer-Meshkov (RM)-like instability occurring on the internal surface of particle rings impinged by divergent blast waves. Despite of the signature spike-bubble instability structure analogous to the hydrodynamic RM instability, the growth of the perturbation amplitude in granular media undergoes an exponential phase followed by a linear phase, markedly differing from the hydrodynamic RM instability, indicating a fundamentally different mechanism. The granular RM-like instability arises from the incipient transverse granular flows induced by the hydrodynamic effects upon the shock interaction. Whereas a substantial perturbation growth is initiated by the ensuing rarefaction dilation when the hydrodynamic effects are minimum. It is the interplay between the localized transverse and radial granular flows that sustains the persistent perturbation growth and drives the morphological changes of instability pattern alongside.
\end{abstract}

\section{Hosted file}

Explosion driven Interfacial instabilities of granular media (AICHe 0404).pdf available at https: //authorea.com/users/309177/articles/440156-explosion-driven-interfacial-instabilities-ofgranular-media 